Published in final edited form as:

Clin Cancer Res. 2019 January 01; 25(1): 290-299. doi:10.1158/1078-0432.CCR-18-2311.

\title{
Arming an Oncolytic Herpes Simplex Virus Type 1 with a Single- chain Fragment Variable Antibody against PD-1 for Experimental Glioblastoma Therapy
}

\author{
Carmela Passaro ${ }^{1}$, Quazim Alayo ${ }^{1}$, Isabella De Laura ${ }^{1}$, John McNulty ${ }^{1}$, Korneel Grauwet ${ }^{1}$, \\ Hirotaka Ito ${ }^{1}$, Vivek Bhaskaran ${ }^{1}$, Marco Mineo ${ }^{1}$, Sean E. Lawler ${ }^{1}$, Khalid Shah ${ }^{1,2}$, Maria C. \\ Speranza $^{1,3}$, William Goins ${ }^{4}$, Eric McLaughlin ${ }^{5}$, Soledad Fernandez ${ }^{5}$, David A. Reardon ${ }^{6}$, \\ Gordon J. Freeman ${ }^{3}$, E. Antonio Chiocca ${ }^{1}$, Hiroshi Nakashima ${ }^{1}$ \\ ${ }^{1}$ Harvey W. Cushing Neuro-oncology Laboratories (HCNL), Department of Neurosurgery, Harvard \\ Medical School and Brigham and Women's Hospital, Boston, Massachusetts \\ ${ }^{2}$ Center for Stem Cell Therapeutics and Imaging (CSTI), Harvard Medical School and Brigham \\ and Women's Hospital, Boston, Massachusetts \\ ${ }^{3}$ Department of Medical Oncology, Dana-Farber Cancer Institute, and Harvard Medical School, \\ Boston, Massachusetts \\ ${ }^{4}$ Department of Microbiology and Molecular Genetics, University of Pittsburgh School of \\ Medicine, Pittsburgh, Pennsylvania \\ ${ }^{5}$ Center for Biostatistics, The Ohio State University, Columbus, Ohio
}

Corresponding Authors: E. Antonio Chiocca, Brigham and Women's Hospital/Harvard Medical School, 75 Francis Street, Boston, MA 02115. Phone: 617-732-6939; Fax: 617-734-8342; EAChiocca@bwh.harvard.edu; and Hiroshi Nakashima,

HNakashima@bwh.harvard.edu.

Authors' Contributions

Conception and design: C. Passaro, S.E. Lawler, D.A. Reardon, G.J. Freeman, E.A. Chiocca, H. Nakashima

Development of methodology: C. Passaro, Q. Alayo, K. Grauwet, V. Bhaskaran, E.A. Chiocca, H. Nakashima

Acquisition of data (provided animals, acquired and managed patients, provided facilities, etc.): C. Passaro, Q. Alayo, I.

DeLaura, H. Ito, M.C. Speranza, W.F. Goins

Analysis and interpretation of data (e.g., statistical analysis, biostatistics, computational analysis): C. Passaro, I. DeLaura, K.

Grauwet, S.E. Lawler, K. Shah, E. McLaughlin, S. Fernandez, D.A. Reardon, G.J. Freeman, E.A. Chiocca

Writing, review, and/or revision of the manuscript: C. Passaro, K. Grauwet, H. Ito, M. Mineo, S.E. Lawler, K. Shah, W.F. Goins,

D.A. Reardon, G.J. Freeman, E.A. Chiocca, H. Nakashima

Administrative, technical, or material support (i.e., reporting or organizing data, constructing databases): M.C. Speranza, W.F.

Goins, E.A. Chiocca, H. Nakashima

Study supervision: S.E. Lawler, E.A. Chiocca, H. Nakashima

Other (helped run the experiments and helped generate the data): J.J. McNulty

Disclosure of Potential Conflicts of Interest

K. Shah is a consultant/advisory board member for AMASA Technologies. W.F. Goins is a consultant/advisory board member for Oncorus INC. D. A. Reardon reports receiving speakers bureau honoraria from and is a consultant/advisory board member for Abbvie, Agenus, Bristol-Myers Squibb, Celldex, EMD Serono, Genentech/Roche, Inovio, Merck, Merck KGaA, Monteris, Novocure, Oncurus, Oxigene, Regeneron, Stemline, and Taiho Oncology Inc. G. J. Freeman holds ownership interest (including patents) in Roche, Merck, Bristol-Myers Squibb, EMD Serono, Boehringer-Ingelheim, AstraZeneca, Dako, and Novartis, and is a consultant/ advisory board member for Roche, Bristol-Myers Squibb, Xios, and Origimed. E.A. Chiocca holds ownership interest (including patents) in DNAtrix, and is a consultant/advisory board member for Advantagene, DNAtrix, and NanoTherapeutics. No potential conflicts of interest were disclosed by the other authors.

Disclaimer

The content is solely the responsibility of the authors and does not necessarily represent the official views of the NIH.

The costs of publication of this article were defrayed in part by the payment of page charges. This article must therefore be hereby marked advertisement in accordance with 18 U.S.C. Section 1734 solely to indicate this fact.

Note: Supplementary data for this article are available at Clinical Cancer Research Online (http://clincancerres.aacrjournals.org/). 
${ }^{6}$ Center for Neuro-Oncology, Dana-Farber Cancer Institute, and Brigham and Women's Hospital, Boston, Massachusetts.

\section{Abstract}

Purpose-Glioblastoma (GBM) is resistant to standard of care. Immune checkpoints inhibitors (such as anti-PD-1 mAbs) efficiently restore antitumor T-cell activity. We engineered a new oncolytic herpes simplex virus (oHSV) expressing a single-chain antibody against PD-1 (scFvPD-1) to evaluate its efficacy in mouse models of GBM.

Experimental Design-NG34scFvPD-1 expresses the human GADD34 gene transcriptionally controlled by the Nestin promoter to allow replication in GBM cells and a scFvPD-1 cDNA transcriptionally controlled by the CMV promoter. ELISA assays were performed to detect binding of scFvPD-1 to mouse and human PD-1. In vitro cytotoxicity and replication assays were performed to measure NG34scFvPD-1 oncolysis, and scFvPD-1 expression and secretion were determined. In vivo survival studies using orthotopic mouse GBM models were performed to evaluate the therapeutic potency of NG34scFvPD-1.

Results-NG34scFvPD-1-infected GBM cells express and secrete scFvPD-1 that binds mouse PD-1. The introduction of the scFvPD-1 sequence in the viral backbone does not alter the oncolytic properties of NG34scFvPD-1. In situ NG34scFvPD-1 treatment improved the survival with a tail of durable survivorship in 2 syngeneic immunocompetent mouse models of GBM. Mice that survived the first GBM challenge rejected the second challenge of GBM when implanted in the contralateral hemisphere. However, this was not true when athymic mice were employed as the recipients of the second challenge, consistent with the need for an intact immune system to obtain a memory response.

Conclusions-NG34scFvPD-1 treatment induces a durable antitumor response in 2 preclinical mouse models of GBM with evidence for antitumor memory.

\section{Introduction}

Glioblastoma (GBM) is the deadliest type of brain tumor. Its annual incidence is 5 per 100,000 adults and it constitutes $15 \%$ of all primary brain tumors and $54 \%$ of all gliomas (1). With the current standard of care, consisting of maximal tumor resection, followed by irradiation and concomitant chemotherapy, the median survival time is 14.6 months after diagnosis and the average 5-year survival rate is less than 5\% (1). GBM's current standardof-care treatments, including surgery and chemoradiotherapy, are not curative $(2,3)$. In recent years, immunotherapy has emerged as a promising approach for cancer treatment with unprecedented responses in certain tumor types. Immunotherapy includes a range of strategies that are aimed to stimulate immune-mediated antitumor responses. Multiple immunotherapeutic strategies have been developed during the last 3 decades, such as antibodies against tumor-specific targets, immune checkpoint inhibitors, vaccines that can be based on dendritic cells, tumor peptides or tumor DNA, oncolytic viruses (OVs), pattern recognition receptor (PRR) agonists, immunostimulatory cytokines, and CAR T cells (4).

Interest in OVs has been increasing since the FDA approved the Herpes virus-based OV (oHSV) talimogene laherparepvec (T-VEC, Imlygic) for use in patients with melanoma (5). 
Moreover, encouraging preclinical results obtained with different oHSVs have led to its testing in several clinical trials in patients with GBM (6-9). OVs are thought to mediate their effects through a dual mechanism involving (i) selective replication and lysis of infected cancer cells, and (ii) induction of host antitumor immunity. The antitumor immune response is a direct consequence of the lytic activity of the virus: OVs can kill cancer cells, most likely by inducing immunogenic cell death followed by the release of tumor-associated antigens (10).

Significant preclinical and clinical results have led to FDA approval of immune checkpoint inhibitors for melanoma, non-small cell lung cancer and other advanced solid tumors (11). The use of mAbs against PD-1 or PD-L1 relieves an inhibitory immune checkpoint, thereby restoring T-cell activation. Therapy with anti-PD-1 has been shown to enhance an antitumor immune response in multiple solid tumors. However, late-phase clinical trials with immune checkpoint blockade against GBM (12) did not result in significant therapeutic benefits (13). Several factors may limit the efficacy of immune checkpoint inhibitors in GBM. These include insufficient tumor immunogenicity, inadequate ability to overcome the immunosuppressive microenvironment, and/or lack of passage of the immune checkpoint inhibitor to cross the blood-brain barrier and disrupt immune checkpoint signaling in situ. Indeed, it has been shown that only a subset of patients with advanced cancers respond to single-agent immune checkpoint blockade (14). Therefore, combinatorial treatments including different immunotherapies may be more effective in GBM and other cancers.

In this work, we hypothesized that the use of an oHSV engineered to express a single-chain fragment variable ( $\mathrm{scFv}$ ) antibody against PD-1 would enhance antitumor immune responses after intratumoral administration. In preclinical immunocompetent models of GBM, this new oHSV extended median survival and importantly induced a memory response against the tumor. This provides support for combining local intratumoral applications of an oncolytic HSV-1 with in situ expression of PD-1 blockade.

\section{Materials and Methods}

\section{Cell lines and cell culture conditions}

Human U251 cells were purchased from ATCC and human U87 $\triangle$ EGFR glioma cells were kindly provided by Webster Cavenee from Ludwig Institute for Cancer Research. 293FT cells were purchased from Thermo Fisher Scientific, and Monkey Vero kidney cells and 293 cells were purchased from ATCC. Human glioma U87 $\mathrm{EGFR,} \mathrm{murine} \mathrm{glioma} \mathrm{GL261N4,}$ and CT2A cell lines were described previously (15). These cells were cultured as monolayers in DMEM (Thermo Fisher Scientific) supplemented with 10\% FBS (Thermo Fisher Scientific), $100 \mathrm{IU} / \mathrm{mL}$ penicillin, and $100 \mu \mathrm{g} / \mathrm{mL}$ streptomycin (Thermo Fisher Scientific) at $37^{\circ} \mathrm{C}$ in a humidified incubator maintained at $5 \% \mathrm{CO}_{2}$. CT2A/PD-L1 cells were generated by transduction with a mouse PD-L1-encoding lentiviral vector as follows: The mPDL1 gene was amplified from Mammalian Gene Collection (MGC) cDNA clone 76638 (GenBank Accession: BC066841; Dana-Farber Cancer Institute, Boston, MA) by PCR with primers (5' ${ }^{\prime}$ CACCATGAGGATATTTGCTGGCATTA- $3^{\prime}$ and $5^{\prime}-$ TTACGTCTCCTCGAATTGTGTATC-3') and cloned into pENTR/D-TOPO (Thermo Fisher Scientific) before Gateway Cloning (Thermo Fisher Scientific) into a pLenti-PGK 
Hygro DEST vector (w530-1; Addgene). The obtained clone was verified by sequencing. Lentivirus was packaged in 293FT cells and infected CT2A. Hygromycin $(50 \mu \mathrm{g} / \mathrm{mL}$; Thermo Fisher Scientific)-resistant clones were selected and PD-L1 gene expression was confirmed by Western blotting with an anti-PD-L1 antibody (10F.9G2, BioLegend).

\section{DNA constructs and HSV packaging}

pcDNA3-scFvPD-1-The scFvPD-1 cDNA encoding heavy- and light-chain variants of PD-1 antibody (clone $332.8 \mathrm{H} 3$; ref. 16) with $3 \times$ linker peptide (GGGGS) was synthesized in GenScript and cloned in pcDNA3 (Invitrogen). This cDNA additionally contains IL2 secretion signal sequences at the $5^{\prime}$ end to facilitate the extracellular secretion and $3^{\prime}$ c-myc and $5 \mathrm{xHis}$ tag sequences for purification and detection.

NG34scFvPD-1 and control NG34-GFP-We previously described the method of HSV construction using the HSVQuik method $(17,18)$. In brief, the scFvPD-1 sequence in pcDNA3-scFvPD-1 DNA was inserted into the SaII sites of a pTransfer-NG34 vector. The resultant shuttle vector was recombined with fHsvQuik1 to obtain fHsvQ1-NG34scFvPD-1 BAC vector. Using this vector, NG34scFvPD-1 virus was generated and packaged in Vero cells. NG34-GFP was generated from pTransfer-NG34 vector with fHsvQuik1. Viral stocks were prepared as described previously $(6,19)$.

\section{Western blot analyses}

Cell lysates (from cells or brain tissue) were prepared in lysis buffer, consisting of 50 $\mathrm{mmol} / \mathrm{L}$ Tris- $\mathrm{HCl}$ (pH 7.4), $150 \mathrm{mmol} / \mathrm{L} \mathrm{NaCl}, 4$ mmol/L EDTA, 0.5\% Triton X-100, PhosSTOP (Sigma) and protease inhibitor cocktail (Roche), sonicated before centrifugation at $20,000 \times g$ for 10 minutes at $4^{\circ} \mathrm{C}$. Supernatants were used for immunoblotting analyses using antibodies against Myc-tag (Cell Signaling Technology, 2272S), GFP (Abgent, AM1009a), and a-tubulin (Sigma, T6074).

\section{scFvPD-1 purification}

HEK293 $\left(1 \times 10^{7}\right.$ cells $)$ were transfected with pcDNA3-scFvPD-1 $(50 \mu \mathrm{g})$ using Lipofectamine 2000 (Thermo Fisher Scientific) in serum-free Expi293 medium (Thermo Fisher Scientific). Medium was collected 48 hours posttransfection, concentrated, and incubated overnight with Ni-NTA agarose beads (Qiagen). scFvPD-1was then purified by gravity-flow chromatography using $200 \mathrm{mmol} / \mathrm{L}$ Imidazole and 10\% glycerol in DPBS and dialyzed in $20 \mathrm{mmol} / \mathrm{L}$ Tris- $\mathrm{HCl} \mathrm{pH}$ 7.5. Each step of the affinity purification was resolved by PAGE and stained with Coomassie Brilliant Blue (to check the purity of the preparation) or immunoblotted using Myc-tag Ab (Cell Signaling Technology, 2272S; to check for scFvPD-1 expression; Supplementary Fig. S1). Protein concentration was determined by Bradford assay.

\section{ELISA assay}

MaxiSorp 96-multiwell plates (BioLegend) were coated with $100 \mu \mathrm{g} /$ well of recombinant mouse (Sino Biological) dissolved in $0.05 \mathrm{~mol} / \mathrm{L}$ carbonate/bicarbonate buffer $(\mathrm{pH} 9.6)$ and incubated overnight at $4{ }^{\circ} \mathrm{C}$. Purified scFvPD-1, full-length anti-PD-1 antibody or 
supernatants from NG34scFvPD-1-infected cells were incubated for 2 hours at room temperature. The binding of the scFvPD-1 or the anti-PD-1 to PD-1 was assayed, using an anti-Myc-HRP (Abcam) or anti-mouse-IgG-HRP (GE Healthcare), respectively. The positive and negative control were prepared by coating the well with the human c-myc peptide (10 $\mu \mathrm{g} / \mathrm{mL}$, Abcam; ab13837) and BSA $(10 \mu \mathrm{g} / \mathrm{mL})$, respectively.

\section{Cytotoxicity assay and viral replication}

For the in vitro cytotoxicity assay, GL261N4, CT2A, U251, and U87 $\mathrm{EGFR}$ cells were seeded onto 96-well plates at densities of 1,000 cells/well and allowed to attach for 24 hours. Cells were then treated with graded concentrations (6 replicates) of NG34 or NG34scFvPD-1 virus. After 6 days, cells were fixed with 50\% vol/vol TCA and stained with $0.4 \% \mathrm{w} / \mathrm{v}$ sulforhodamine $\mathrm{B}$ in $1 \% \mathrm{vol} / \mathrm{vol}$ acetic acid (20). The percentages of viable cells after treatment were calculated by comparison with control noninfected cells, and the median lethal dose $\left(\mathrm{LD}_{50}\right)$ was determined by a dose-response curve (variable slope, 4 parameters) using GraphPad Prism (GraphPad Software). For in vitro viral replication, GL261N4, CT2A, U251, and U87 $\triangle$ EGFR cells (100,000 cells) were seeded onto 6-well plates, 24 hours before infection. Cells were then treated with NG34 or NG34scFvPD-1 virus $(0.003$ or $0.03 \mathrm{pfu} / \mathrm{cell})$. After 72 hours, cells and media were collected and cells were disrupted by 3 cycles of freeze-thaw in dry ice-chilled ethanol, centrifuged at $300 \times g$ for 5 minutes, and supernatants were collected for viral titration on Vero cells (6).

\section{Animal use}

All experimental procedures using animals were carried out under an animal protocol reviewed and approved by the Harvard Center for Comparative Medicine (HCCM) and the BWH Center for Comparative Medicine insttutional Animal Care and Use Committee. Female gender-matched tumors and mice were utilized for in vivo studies. Six- to 8-weekold C57Bl/6J strain mice were purchased from Jackson Laboratories and 6- to 8-week-old athymic nude strain mice were purchased from Envigo. For in vivo survival studies, dissociated GL261N4, CT2A, or CT2A/PD-L1 (100,000 cells in $5 \mathrm{~mL}$ of HBSS for GL261N4 and 400,000 cells in $5 \mathrm{~mL}$ of HBSS for CT2A and CT2A/PD-L1) were injected intracerebrally at stereotactic coordinates [ventral $3.5-\mathrm{mm}$, rostral $0.5-\mathrm{mm}$, and right lateral 2.0-mm from the bregma using a stereotaxic apparatus (David Kopf Instruments)] to establish the tumor in the brains of mice. Intratumoral injections of oHSV were performed at the indicated timing and doses described in Figs. 4 and 6B. Intraperitoneal injection of the anti-PD-1 antibody (clone 332.8H3, $200 \mu \mathrm{g} /$ mouse) were performed in CT2A or CT2A/ PDL1 starting at day 7 after tumor implantation every 3 days for a total of 4 injections.

For in vivo scFvPD-1, ICP4, and gD expression, GL261N4 and U87 $\triangle \mathrm{EGFR}$ cells were implanted in $\mathrm{C} 57 \mathrm{Bl} / 6 \mathrm{~J}$ and nude athymic mice and then NG34scFvPD-1 was intratumorally administered $\left(1.5 \times 10^{6} \mathrm{pfu} /\right.$ mouse and $5 \times 10^{5} \mathrm{pfu} /$ mouse for $\mathrm{C} 57 \mathrm{Bl} / 6 \mathrm{~J}$ and athymic nude mice, respectively) as described above. The mice were then sacrificed at different time points. For in vivo viral replication, mice were sacrificed 48 hours after NG34scFvPD-1 treatment and brains were harvested and mechanically dissociated in DMEM containing 2\% FBS. Cells were disrupted by 3 freeze-thaw cycles, centrifuged at $300 \times g$ for 5 minutes, and supernatants were collected for viral titration on Vero cells. 


\section{RNA isolation and real-time PCR}

Total RNA was extracted from harvested tissues using TRIzol reagent (Thermo Fisher Scientific) and treated with RNase-free DNase (Qiagen) according to the manufacturer's instructions. One microgram of total RNA was reverse-transcribed using iScript cDNA synthesis kit (Bio-Rad Laboratories). Real-time PCR was carried out using Power SYBR Green (Applied Biosystems). The mRNA copy number was calculated on the basis of standard curves made with known concentrations of the target templates (scFvPD-1, ICP-4, and $\mathrm{gD}$ ). The sequences of the primers used for the reaction are the following:

scFvPD-1: forward, $5^{\prime}$-CTACATCCACCCCAGCACAG-3' ${ }^{\prime}$ scFvPD-1 reverse, $5^{\prime}$ CGAACAGGTAGGTGCCCTTT-3' ${ }^{\prime}$; ICP4: forward, $5^{\prime}$-GGCCTGCTTCCGGATCTC-3'; ICP4 reverse, $5^{\prime}$-GGTGATGAAGGAGCTGCTGTT-3'; gD forward, $5^{\prime}$ ACGGTTTACTACGCCGTGTT-3'; gD reverse, 5' ${ }^{\prime}$-TGTAGGGTTGTTTCCGGACG-3' .

\section{Statistical analysis}

Statistical analysis was performed with Prism 6 (GraphPad Software). $P$ values were calculated with paired $t$ test or repeated measure one-way ANOVA corrected for multiple comparisons unless otherwise indicated. $P<0.05$ was considered significant. Kaplan-Meier analysis was performed using log-rank Mantel-Cox test corrected for multiple comparisons (Holm post test).

\section{Results}

\section{An anti-PD1 single-chain fragment variable antibody efficiently binds the mouse PD-1 protein}

To generate a scFv antibody against mouse PD-1, the variable regions of heavy and light chains were sequenced from the hybridoma clone $332.8 \mathrm{H} 3$ and this codon-optimized cDNA was synthesized and cloned in an expression vector $(16,17)$. We tested whether this $\mathrm{scFv}$ antibody (scFvPD-1) could bind mouse PD-1 protein. The purified scFvPD-1 recombinant protein and the full-length antibody $(332.8 \mathrm{H} 3 \mathrm{IgG})$ were subjected to an ELISA to analyze binding to mouse PD-1 (Fig. 1). Both the scFvPD-1 and full-length aPD-1 antibody showed similar dose-dependent binding to PD-1 (Fig. 1). This result justified the use of scFvPD-1 against mouse GBMs that express mouse PD-L1.

\section{Glioma cells infected with NG34scFvPD-1 produce and secrete scFvPD-1}

We next engineered an oHSV encoding the scFvPD-1 cDNA under the transcriptional control of a CMV IE promoter. This oHSV (NG34scFvPD-1) is characterized by insertion of a human $G A D D 34$ gene in the viral gene locus that encodes for ICP6 under the control of a Nestin-hsp68 hybrid promoter to promote cytotoxicity in GBM cells (15). NG34scFvPD-1 also was engineered with deletions of the viral ICP6 and ICP34.5 coding gene loci (Supplementary Fig. S2A; refs. 15, 17). We confirmed in producer Vero cells that selected viral clones expressed the myc-tagged $25 \mathrm{kDa}$ scFvPD-1 protein in cells and supernatants (Supplementary Fig. S2B and S2C). One of the clones (clone 5.6) was chosen for further experiments because it showed the highest production and secretion of the myc-tagged scFvPD-1 protein. We next tested the ability of NG34scFvPD-1 to produce and secrete 
scFvPD-1 in 2 different mouse GBM cells (GL261N4 and CT2A). As shown in Fig. 2A, both cells produced and secreted scFvPD-1 after infection with NG34scFvPD-1. The same was true for human GBM cells (data not shown). To quantify the amount of scFvPD-1, we infected mouse GL261N4 and CT2A and human U251 and U87 $\triangle E$ EGFR cells with NG34scFvPD-1 (1 pfu/cell) and assayed scFvPD-1 amount in the supernatant 24 hours later. GL261N4 cells (Fig. 2B) produced a similar amount of scFvPD-1 compared with infected human cells (Fig. 2C). CT2A produced 4 times less scFvPD-1 compared with all the other cell lines (Fig. 2B), likely due to lower infectivity by the oHSV, based on reduced percent of GFP-positive CT2A cells compared with GL261NA 24 hours after infection (Supplementary Fig. S2D). These results thus indicated that NG34scFvPD-1 infection led to production and secretion of scFvPD-1 in infected GBM cells.

\section{ScFvPD-1 does not alter oncolytic properties of the oHSV}

Next, we tested whether the expression of the scFvPD-1 altered the oncolytic properties of the NG34 oHSV. Mouse GBM cells (GL261N4 cells and CT2A) and human GBM cells (U251 and U87 $\triangle$ EGFR) were incubated with increasing doses of both NG34scFvPD-1 and the parental virus NG34. There was no significant difference in the $\mathrm{LD}_{50}$ between the 2 oHSVs (Fig. 3A). We also tested whether virus replication was affected by the oHSV genome modification. This showed that there was no significant difference in viral replication between NG34scFvPD-1 and parental NG34 (Fig. 3B). We thus conclude that insertion of scFvPD-1 sequences or its expression did not significantly alter the infectivity and the oncolytic properties of NG34scFvPD-1.

\section{NG34scFvPD-1 improves survival of immunocompetent, but not athymic mice with orthotopic GBMs}

We next sought to determine whether NG34scFvPD-1 was an effective therapeutic in mouse models of GBM. We implanted GL261N4 cells in the right hemisphere of the brain of immunocompetent C57B1/6 mice. Seven days after tumor implantation, when tumors had all achieved volumes that were similar as measured by MRI (data not shown), we stereotactically injected into the tumor NG34scFvPD-1 or the parental NG34. There was a significant prolongation of the median survival time in mice treated with NG34scFvPD-1 compared with untreated (CTRL) mice (69 vs. 22 days, respectively; Fig. 4A).

NG34scFvPD-1 also doubled the median survival time of mice when compared with mice treated with parental virus NG34 (69 vs. 36.5 days, respectively).

Both oHSVs had a cohort of mice that survived up to 120 days, at which time the experiment was terminated. We tested whether these surviving mice would tolerate a second challenge with the same tumor cells in the contralateral hemisphere. Tumor-naïve agematched mice were used as controls. All 3 surviving mice whose GBMs had been treated with NG34scFvPD-1 and 2 of 3 surviving mice whose GBMs had been treated with NG34 survived this tumor rechallenge while control naïve mice did not (Fig. 4B). These data thus showed that mice with murine GBM that had been successfully treated with either oHSV were also protected from subsequent tumor rechallenge. 
We also wanted to determine whether the NG34 or NG34scFvPD-1 survival effect on GBM were dependent on an antitumor immune response. We thus utilized athymic mice with orthotopic mouse GL261N4 GBMs. Neither NG34 nor NG34scFvPD-1 treatment led to survival extension in these mice compared with the vehicle control (Fig. 4C). Taken together, these data suggest that the treatment benefit of these oHSVs in mouse GBM models required a T-cell immune response.

\section{Limited oHSV replication and scFvPD-1 production in mouse GL261N4 GBMs in vivo}

The above experiments led us to determine the time course of scFvPD-1 production and oHSV gene expression in mouse GL261N4 GBMs in vivo. Immunocompetent C57B1/6 mice with G1261N4 GBMs were treated with NG34scFvPD-1 and tumors were harvested at different time points to assay for scFvPD-1 expression. There was evidence of scFvPD-1 mRNA in the brain of treated mice 16 hours postinfection, but the levels were significantly reduced by 36 hours and had essentially disappeared by 60 hours (Fig. 5A). This peak of scFvPd-1 transcript production followed by the observed reduction and disappearance of the transcript paralleled the mRNA copy number of the HSV-1 immediate early gene ICP4 and late gene $g D$ (Fig. 5B). This comparatively short period of expression of viral genes thus suggested that the oHSV did not replicate well in mouse GL261N4 GBM cells in vivo.

\section{NG34scFvPD-1 replicates and produces ScFvPD-1 in human GBM cells in mice}

We wanted to ensure that the oHSV replicated and produced scFvPD-1 in human GBMs in vivo. Athymic mice were injected intracranially with human U87 $\triangle$ EGFR cells or mouse GL261N4 cells and 7 days later were inoculated with NG34scFvPD-1. We assayed mRNA expression in brains harvested 48 hours later. In 2 of 3 mice challenged with U87 $\triangle E G F R$ cells, there was a relatively elevated mRNA copy number for both ICP4 and gD when compared with mice challenged with GL261N4 cells (Supplementary Fig. S3A). scFvPD1 mRNA transcript was also detected in U87 There was also active oHSV harvested from the brains of mice with U87 $\mathrm{EGFR}$ tumors, but none from the brains of mice with GL261N4 tumors (Supplementary Fig. S3B). Therefore, NG34scFvPD-1 can replicate and express in human GBM cells. In mouse GBM cells, there is an initial infection and expression of oHSV genes and of scFvPD-1 but by $48-60$ hours (Fig. 5; Supplementary Fig. S3), the virus and its transcripts cannot be detected.

\section{NG34scFvPD-1 can also induce a memory response in the CT2A immunocompetent model of GBM}

To determine whether NG34scFvPD-1 led to therapeutic efficacy in a second model of mouse GBM, we intracranially injected C57Bl/6 mice with mouse CT2A GBM cells. First, we investigated whether CT2A GBMs were responsive to anti-PD-1. However, this was not the case as shown in Fig. 6A. We then overexpressed the murine PD-1 ligand, PD-L1, in $\mathrm{CT} 2 \mathrm{~A}$ cells and repeated the experiment. With CT2A/PD-L1 GBMs, there was now significant therapeutic sensitivity to anti-PD-1 compared with isotype antibody (Fig. 6B). In further experiments, we thus utilized CT2A/PD-L1 GBMs that were injected with NG34scFvPD-1 or NG34. In vitro, infection and replication of either oHSV was relatively inefficient (see Fig. 3) and the same was true in vivo (data not shown). Although neither oHSV led to a significant increase in median survival, 2 of 12 mice treated with 
NG34scFvPD-1 did survive for the duration of the experiment (120 days; Fig. 6C). These 2 surviving mice rejected a rechallenge with CT2A/PD-L1 GBMs (Fig. 6D). These findings thus showed that expression of scFvPD-1 from the oHSV led to mouse survival in a second GBM model with establishment of an antitumor memory response.

\section{Discussion}

There has been high interest in the use of immunotherapies, such as immune checkpoint inhibitors, against cancers including GBM. However, systemic single immune checkpoint inhibition has not shown to be effective in a recent randomized phase III clinical trial in GBM (13). While there may be multiple factors responsible for this, one major issue may be related to the need for combinatorial therapies to overcome multiple mechanisms of immune-evasion (21-23). Another factor may relate to the need for the PD-1 antibody to interact with PD-L1 in the GBM microenvironment and the unique problem of therapy access to this tumor due to the blood-brain barrier (BBB), which may not allow access to large molecules. We thus reasoned that "arming" an oncolytic virus, such as oHSV, with an immune checkpoint inhibitor antibody would provide both a combinatorial immunotherapy and intratumoral delivery/expression of the antibody. Here we show that an oncolytic HSV1 armed with a scFvPD-1 retained baseline oncolytic capacity and produced and secreted a scFvPD-1 in infected mouse and human GBM cells. In the relatively immunogenic mouse GL261nectin-1 GBM model, both NG34scFvPD1 and parental NG34 oHSV, effectively increased survival of mice but the inclusion of the scFvPD-1 did not increase survival. The relative lack of NG34scFvPD1 efficacy compared with NG34 was due to the inherent poor replication and thus low level of production of scFvPD1 by oHSV in mouse GBMs in vivo. In a second mouse model of GBM (CT2A), which is less sensitive to anti-PD-1 therapy and less immunogenic compared with GL261 GBMs, NG34 was not effective compared with control, but NG34scFvPD1 did lead to a low percentage (17\%) of mice with long-term responses. In both GL261N4 and CT2A models, observed responses required T-cell activity against infected GBMs and surviving mice developed memory against further GBM challenge. Therefore, we conclude that NG34scFvPD1 is a therapeutically valid option, yet full analysis is limited by the challenge of mouse GBM models not allowing for high levels of in vivo replication of oHSV and thus high levels of scFvPD1 production.

The oHSV Talimogene laherparepvec remains the only FDA-approved therapy for cancer (advanced melanoma). In human melanoma patients, elevated PD-L1 protein expression and IFN $\gamma$ gene expression following TVEC treatment were also observed, suggesting CD8 ${ }^{+} \mathrm{T}-$ cell activation (24) and providing the biologic justification to add an anti-PD-1 inhibitor. For this purpose, we armed a different oHSV (NG34) that has selectivity for GBM with a singlechain variable fragment antibody against PD-1. We chose anti-PD-1 over another immune checkpoint inhibitor (anti-CTLA-4) for several reasons: (i) the production of the $\mathrm{scFv}$ antibody is limited at the site of infection and therefore the presence of PD-L1 on tumor and immune cells makes the PD-1 pathway a more appealing target; (ii) 2 independent studies in a mouse model of GBM showed that compared with other immune checkpoint inhibitors, CTLA-4 blockade was the least effective at antitumor responses and survival benefit and mainly functions at lymph nodes where CD4 and CD8 T cells are activated upon antigen presentation, rather than tumor bed itself (refs. 16, 25, 26); (iii) in a preclinical melanoma 
study, the use of a Myxoma virus secreting a soluble form of PD-1 improved mouse survival, showing minor side effects compared with the combination of a Myxoma virus with systemic anti-PD-1 therapy (27).

One possible concern was whether scFvPD1 would bind to PD-1. Our studies show that purified scFvPD-1 did bind PD-L1 of both mouse and human origin. Mouse and human PD-1 sequences share approximately $60 \%$ amino acid identity overall, with higher conservation in these extracellular regions ( $65 \%$ identity; ref. 28$)$. Therefore, the binding to PD-1 from either species was expected. We were also able to show that arming NG34 with scFvPD-1 did not hinder oHSV replication and cytolytic activity against mouse and human GBM cells and that the scFvPD-1 was expressed and secreted from infected GBM cells in vitro.

Evaluating oHSV activity in vivo is complicated by the relative lack of infectivity and replication of oHSV in mouse GBM cells and the relative nonpermissivity of immunocompetent C57B1/6 mice to HSV infection (29-31). Unfortunately, most of the wellestablished GBM murine models are derived from C57B1/6 mice (such as CT2A and GL261), thus limiting the evaluation of oHSV effects (32). We attempted to address this issue by engineering GL261 GBM cells with nectin-1, the major entry receptor for HSV1. In vitro, this resulted in enhanced infectivity that approximated that in some human GBM cells (data not shown). GL261N4 cells may have some degree of enhanced immunogenicity but all mice that were challenged in their brains with these GBM cells do perish, underscoring that the implanted tumors are not rejected and are lethal to mice. In vivo we found that NG34scFvPD-1 significantly prolonged animal survival compared with control mice (69 vs. 22 days, respectively). Although NG34scFvPD-1 almost doubled mice survival compared with parental virus NG34 (69 vs. 36.5 days, respectively), this comparison ended up not being statistically significant due to sample size and the presence of long-term surviving mice in both NG34 and NG34scFvPD-1-treated mice.

Another possible reason for the short-term effect on survival of the scFvPD-1-expressing virus relates to the in vivo temporal kinetics of scFvPD-1 expression. In NG34scFvPD-1treated mice, mRNA levels for the transgene as well as for viral genes showed an initial burst of expression (16-hour time point), but then rapidly declined. This strongly suggests that the oHSV initially infected some GBM cells, but that there was very little further infection by replicating progeny viruses, likely due to the aforementioned nonpermissivity of C57BL/6 mice, but also due to the lack of effective replication in vivo because even in athymic mice, the oHSV had no effect. The difference in replication of the oHSV in GL261N4 cells in vitro versus in vivo may be due to the mouse strain (C57Bl/6) known be impervious to HSV infection (33). These results though are in general agreement with another study performed by Cheema and colleagues, in which they showed that an oHSV expressing mIL12 (called G47 $\Delta$-mIL12) also did not replicate well in the mouse 005 cell model (C57Bl/6 background; ref. 34). Despite this replicative defect, G47 $\Delta$-mIL12 significantly enhanced survival compared with both untreated mice and mice treated with the parental virus. This can be explained by the strong activity of mIL12 on IFN $\gamma$ release (still present 6 days after treatment) and to the modest antitumor effect of the parental virus (34). 
The in vivo decrease of scFvPD-1 expression may also result from methylation of the heterologous CMV promoter (35). Utilizing a homologous promoter such as the HSV-1 IE4/5 promoter could have resulted in increased expression of the transgene. Improvement in scFvPD-1 secretion from infected murine cells may also be tested by utilizing a different secretory motif in the construct, such as mouse Ig kappa light chain V-III region or CD33.

In the CT2A mouse GBM model, we also could show that NG34scFvPD1 led to mice with apparent long-term responses. Immunotherapy strategies have in general shown to lead to a low percentage of responders that exhibit highly durable tumor responses, resulting in a plateau in the tail of the survival curve (36). The results obtained in our study suggest that the novel oncolytic virus NG34scFvPD-1 does act as an immunotherapeutic agent in our preclinical model of GBM. However, this may not be the case with all OVs, because a different OV armed with both anti-CTLA4 and anti-PD-L1 led to an improvement of survival, but no durable response (37). In both CT2A and GL261N4 models, the most significant finding was that long-term surviving mice developed effective long-term memory responses against rechallenge with the same mouse GBM. This suggests that in mouse GBM models in vivo successful oHSV effects requires effective T-cell-mediated immune responses. This is in contrast to the analysis of oHSVs in human GBM models in athymic mice where effects are almost all due to direct oHSV lytic/replicative effects. In fact, NG34 improves the survival of athymic mice intracranially implanted with human GBM cells (15). We are thus still limited in our capacity to fully analyze the multimodal therapeutic effects of oHSVs, preclinically in mice. One possible solution may be testing oHSVs in humanized mouse models, to analyze both oHSVinduced replication/lysis and T-cell antitumor immune responses (38). In this context, oHSV will efficiently infect, replicate, express scFvPD-1, and lyse tumor cells while human immune cells will infiltrate the tumor microenvironment to possibly mount an antitumor response. Immune-suppressive cells in the GBM microenvironment, such as Tregs, TAMs, MDSCs, and microglia, express the PD-1 and CTLA-4 ligands PD-L1, PD-L2, CD80, and CD86, and are also targets of immune checkpoint inhibitors (39). Expression of anti-PD-1 in situ combined with other signal costimulatory agonist and coinhibitory antagonist antibodies (i.e. CD28, CD40, OX40, antiCTLA4, anti-LAG3), may further improve immune response against GBMs.

In conclusion, NG34scFvPD1 induces both a durable and a memory response in preclinical models of GBM and is a promising agent for future testing in a clinical setting.

\section{Supplementary Material}

Refer to Web version on PubMed Central for supplementary material.

\section{Acknowledgments}

Research reported in this publication was supported by the NCI of the NIH under award numbers 2P01CA163205, CA069246-20, and P50CA165962 (to E.A. Chiocca) and P50CA101942 and P01AI056299 (to G.J. Freeman). This work was also supported the American Brain Tumor Association (to C. Passaro, Basic Research Fellowship). We also would like to recognize Dr. Xandra O. Breakefield (MGH) for critical reading of the manuscript. 


\section{References}

1. Neagu MR, Reardon DA. An update on the role of immunotherapy and vaccine strategies for primary brain tumors. Curr Treat Options Oncol 2015;16:54. [PubMed: 26454859]

2. Stupp R, Mason WP, van den Bent MJ, Weller M, Fisher B, Taphoorn MJB, et al. Radiotherapy plus concomitant and adjuvant temozolomide for glioblastoma. N Engl J Med 2005;352:987-96. [PubMed: 15758009]

3. Kaufmann JK, Chiocca EA. Glioma virus therapies between bench and bedside. Neuro-Oncol 2014;16:334-51. [PubMed: 24470549]

4. Hoos A Development of immuno-oncology drugs - from CTLA4 to PD1 to the next generations. Nat Rev Drug Discov 2016;15:235-47. [PubMed: 26965203]

5. Ledford H Cancer-fighting viruses win approval. Nature 2015;526:622-3. [PubMed: 26511559]

6. Kambara H, Okano H, Chiocca EA, Saeki Y. An oncolytic HSV-1 mutant expressing ICP34.5 under control of a nestin promoter increases survival of animals even when symptomatic from a brain tumor. Cancer Res 2005;65:2832-9. [PubMed: 15805284]

7. Todo T, Tanaka M, Ito M, Ito H, Ino Y. Clinical trials of a third-generation recombinant oncolytic HSV-1 in recurrent glioblastoma and olfactory neuroblastoma patients. Neuro-Oncol 2014;16:iii51.

8. Markert JM, Razdan SN, Kuo H-C, Cantor A, Knoll A, Karrasch M, et al. A phase 1 trial of oncolytic HSV-1, G207, given in combination with radiation for recurrent GBM demonstrates safety and radiographic responses. Mol Ther J Am Soc Gene Ther 2014;22:1048-55.

9. Markert JM, Medlock MD, Rabkin SD, Gillespie GY, Todo T, Hunter WD, et al. Conditionally replicating herpes simplex virus mutant, G207 for the treatment of malignant glioma: results of a phase I trial. Gene Ther 2000;7:867-74. [PubMed: 10845725]

10. Kaufman HL, Kohlhapp FJ, Zloza A. Oncolytic viruses: a new class of immunotherapy drugs. Nat Rev Drug Discov 2015;14:642-62. [PubMed: 26323545]

11. Cully M Combinations with checkpoint inhibitors at wavefront of cancer immunotherapy. Nat Rev Drug Discov 2015;14:374-5. [PubMed: 26027531]

12. Reardon DA, Freeman G, Wu C, Chiocca EA, Wucherpfennig KW, Wen PY, et al. Immunotherapy advances for glioblastoma. Neuro-Oncol 2014;16: 1441-58. [PubMed: 25190673]

13. Filley AC, Henriquez M, Dey M. Recurrent glioma clinical trial, CheckMate-143: the game is not over yet. Oncotarget 2017;8:91779-94. [PubMed: 29207684]

14. Lim M, Weller M, Chiocca EA. Current state of immune-based therapies for glioblastoma. Am Soc Clin Oncol Educ Book 2016;35: e132-139. [PubMed: 27249715]

15. Nakashima H, Nguyen T, Kasai K, Passaro C, Ito H, Goins WF, et al. Toxicity and efficacy of a novel GADD34-expressing oncolytic HSV-1 for the treatment of experimental glioblastoma. Clin Cancer Res 2018;24:2574-84. [PubMed: 29511029]

16. Reardon DA, Gokhale PC, Klein SR, Ligon KL, Rodig SJ, Ramkissoon SH, et al. Glioblastoma eradication following immune checkpoint blockade in an orthotopic, immunocompetent model. Cancer Immunol Res 2016;4:124-35. [PubMed: 26546453]

17. Nakashima H, Chiocca EA. Modification of HSV-1 to an oncolytic virus. Methods Mol Biol 2014;1144:117-27. [PubMed: 24671680]

18. Terada K, Wakimoto H, Tyminski E, Chiocca EA, Saeki Y. Development of a rapid method to generate multiple oncolytic HSV vectors and their in vivo evaluation using syngeneic mouse tumor models. Gene Ther 2006;13: 705-14. [PubMed: 16421599]

19. Nakashima H, Kaufmann JK, Wang P-Y, Nguyen T, Speranza M-C, Kasai K, et al. Histone deacetylase 6 inhibition enhances oncolytic viral replication in glioma. J Clin Invest 2015;125:4269-80. [PubMed: 26524593]

20. Vichai V, Kirtikara K. Sulforhodamine B colorimetric assay for cytotoxicity screening. Nat Protoc 2006;1:1112-6. [PubMed: 17406391]

21. Mirzaei R, Sarkar S, Yong VW. T cell exhaustion in glioblastoma: intricacies of immune checkpoints. Trends Immunol 2017;38:104-15. [PubMed: 27964820]

22. Boussiotis VA, Charest A. Immunotherapies for malignant glioma. Oncogene 2018;37:1121-41. [PubMed: 29242608] 
23. Lim M, Xia Y, Bettegowda C, Weller M. Current state of immunotherapy for glioblastoma. Nat Rev Clin Oncol 2018;15:422-42. [PubMed: 29643471]

24. Ribas A, Dummer R, Puzanov I, Vander Walde A, Andtbacka RHI, Michielin O, et al. Oncolytic virotherapy promotes intratumoral $\mathrm{T}$ cell infiltration and improves anti-PD-1 immunotherapy. Cell 2017;170:1109-1119.e10. [PubMed: 28886381]

25. Buchbinder EI, Desai A. CTLA-4 and PD-1 pathways. Am J Clin Oncol 2016;39:98-106. [PubMed: 26558876]

26. Wainwright DA, Chang AL, Dey M, Balyasnikova IV, Kim CK, Tobias A, et al. Durable therapeutic efficacy utilizing combinatorial blockade against IDO, CTLA-4, and PD-L1 in mice with brain tumors. Clin Cancer Res 2014;20:5290-301. [PubMed: 24691018]

27. Bartee MY, Dunlap KM, Bartee E. Tumor-localized secretion of soluble PD1 enhances oncolytic virotherapy. Cancer Res 2017;77:2952-63 [PubMed: 28314785]

28. Cheng X, Veverka V, Radhakrishnan A, Waters LC, Muskett FW, Morgan SH, et al. Structure and interactions of the human programmed cell death 1 receptor. J Biol Chem 2013;288:11771-85. [PubMed: 23417675]

29. Abghari SZ, Stulting RD, Nigida SM, Downer DN, Nahmias AJ. Comparative replication of HSV-1 in BALB/c and C57BL/6 mouse embryo fibroblasts in vitro. Invest Ophthalmol Vis Sci 1986;27:909-14. [PubMed: 3011703]

30. Kastrukoff LF, Lau AS, Thomas EE. The effect of mouse strain on herpes simplex virus type 1 (HSV-1) infection of the central nervous system (CNS). Herpesviridae 2012;3:4. [PubMed: 22449238]

31. Wintergerst U, Gangemi JD, Kern ER, Whitley RJ, Chatterjee S. Differential effect of recombinant human and mouse interferons on replication of herpes simplex virus type 1 in mouse cells. Antiviral Res 1996;32:27-34. [PubMed: 8863993]

32. Oh T, Fakurnejad S, Sayegh ET, Clark AJ, Ivan ME, Sun MZ, et al. Immunocompetent murine models for the study of glioblastoma immunotherapy. J Transl Med 2014;12:107. [PubMed: 24779345]

33. Kastrukoff LF, Lau AS, Takei F, Carbone FR, Scalzo AA. A NK complex-linked locus restricts the spread of herpes simplex virus type 1 in the brains of C57BL/6 mice. Immunol Cell Biol 2015; 93:877-84. [PubMed: 25971711]

34. Cheema TA, Wakimoto H, Fecci PE, Ning J, Kuroda T, Jeyaretna DS, et al. Multifaceted oncolytic virus therapy for glioblastoma in an immunocompetent cancer stem cell model. Proc Natl Acad Sci U S A 2013; 110:12006-11. [PubMed: 23754388]

35. Bailey LA, Hatton D, Field R, Dickson AJ. Determination of Chinese hamster ovary cell line stability and recombinant antibody expression during long-term culture. Biotechnol Bioeng 2012;109:2093-103. [PubMed: 22896849]

36. Ribas A, Hersey P, Middleton MR, Gogas H, Flaherty KT, Sondak VK, et al. New challenges in endpoints for drug development in advanced melanoma. Clin Cancer Res 2012;18:336-41. [PubMed: 22142824]

37. Engeland CE, Grossardt C, Veinalde R, Bossow S, Lutz D, Kaufmann JK, et al. CTLA-4 and PDL1 checkpoint blockade enhances oncolytic measles virus therapy. Mol Ther J Am Soc Gene Ther 2014;22: 1949-59.

38. Walsh N, Kenney L, Jangalwe S, Aryee K-E, Greiner DL, Brehm MA, et al. Humanized mouse models of clinical disease. Annu Rev Pathol 2017;12: 187-215. [PubMed: 27959627]

39. Cassetta L, Kitamura T. Targeting tumor-associated macrophages as a potential strategy to enhance the response to immune checkpoint inhibitors. Front Cell Dev Biol 2018;6:38. [PubMed: 29670880] 


\section{Translational Relevance}

Glioblastoma (GBM) is an incurable cancer and standardof-care therapies have been minimally effective. Oncolytic viruses (OVs) and immune checkpoint PD-1 inhibitors have become standard-of-care immunotherapies against some cancers and are also being tested in clinical trials for GBM. Here, we test the efficacy of a novel oncolytic virus (NG34scFvPD-1) based on herpes simplex virus type-1 (HSV-1), which also expresses a single-chain fragment variable antibody against PD-1 (scFvPD-1). In vitro,

NG34scFvPD-1 infects mouse and human GBM cells and expresses the scFvPD-1, which is secreted into the culture medium. In vivo, NG34scFvPD-1 leads to durable and longterm survival responses in the GL261 and CT2A immunocompetent mouse GBM models. These studies thus provide justification for further exploration of this novel OV for cancer therapy. 


\section{mPD-1 ELISA}

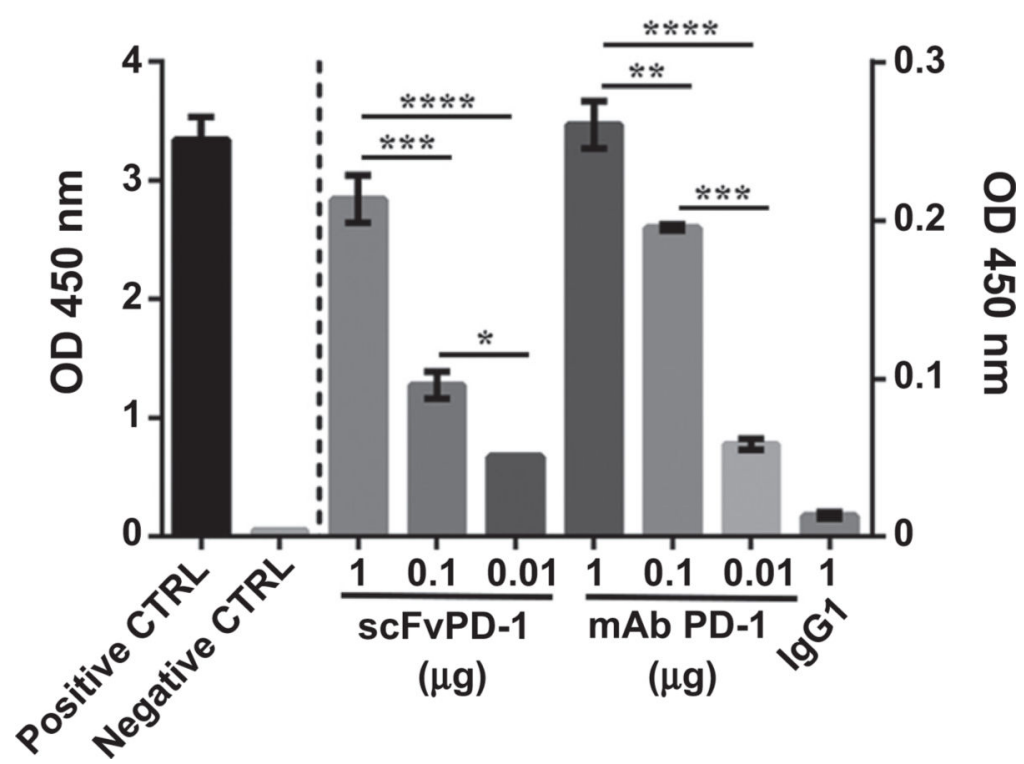

Figure 1.

In vitro evaluation of the ability of scFvPD-1 to bind PD-1. Specific interaction of the purified scFvPD-1 (scFv) and PD-1 mAbs (332.8H3) with murine PD-1 (mPD-1) was quantified by ELISA with a secondary antibody HRP-conjugated anti-myc or anti-mouse IgG1, respectively. Positive control (CTRL): coating with human c-myc peptide. Negative CTRL, coating with BSA. Absorbance (y-axis) represents optical density at $450 \mathrm{~nm}$ corrected by subtract reading at 570nm. Error bar shows the SEM. Group means were compared by one-way ANOVA (Tukey multiple comparisons test), where *, **, ***, and $* * * *$ indicate $P<0.05,0.01,0.001$, and 0.0001 , respectively $(n=3)$. The experiment shown is representative of experiments that were performed at least 3 times. 


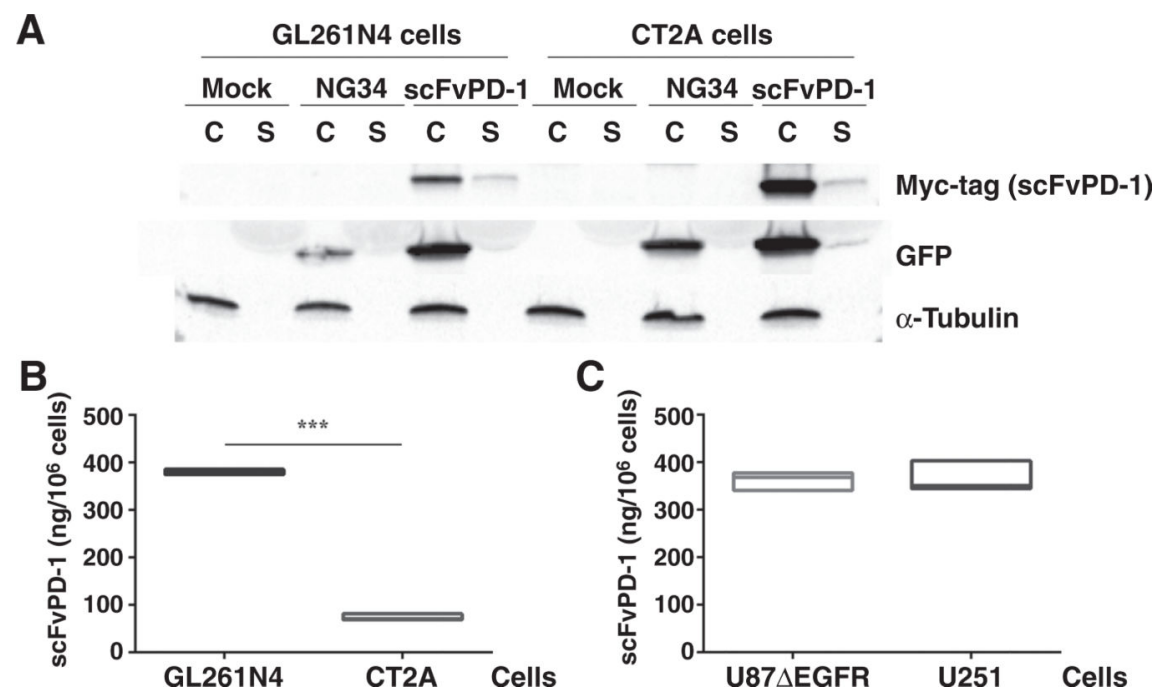

Figure 2.

In vitro evaluation of scFvPD-1 production after oHSV infection. A, GL261N4 and CT2A cells were infected with 1 MOI of NG34scFvPD-1 or NG34 virus. After 24 hours, intracellular proteins (c) and secreted proteins (s) were loaded on a polyacrylamide gel and assessed for scFvPD-1 production (myc-tagged) by Western blot analysis. GFP was used to monitor infection. a-Tubulin was used to check contamination of supernatant with intracellular proteins. c, intracellular protein (loaded $20 \mu \mathrm{g}$ ); s, supernatant (loaded $25 \mu \mathrm{L}$ ). The Western blot is representative of 3 different experiments. B, Supernatant from $10^{6}$ GL261N4 and CT2A cells infected with 1 MOI of NG34scFvPD-1 for 24 hours was assayed for production of scFvPD-1 and its ability to bind mouse PD-1 using an ELISA assay. The amount of scFvPD-1 produced was calculated on the basis of a standard curve generated with purified scFvPD-1. Error bar shows the SEM. Group means were compared by unpaired $t$ test with Welch correction where $* * *$ indicates $P<0.001(n=3)$. C, Supernatants from $10^{6}$ U87 $\triangle$ EGFR and U251 cells were infected with NG34scFvPD-1 (MOI = 1) for 24 hours and then assayed for the production of scFvPD-1 and its binding to mouse PD-1 using an ELISA assay. The amount of scFvPD-1 produced was calculated on the basis of a standard curve generated with purified scFvPD-1. The experiment shown is representative of experiments that were performed at least 3 times. 
A

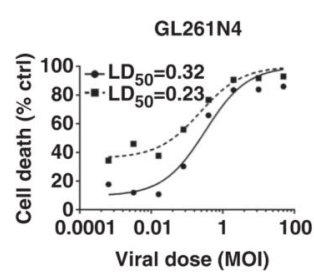

CT2A

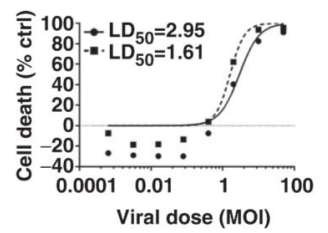

U251

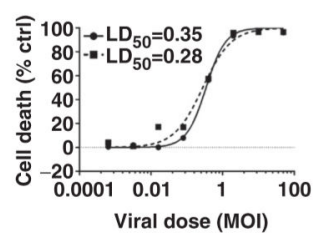

U87 $\triangle E G F R$

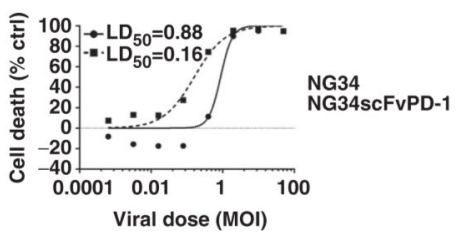

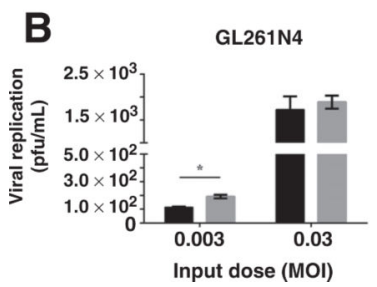
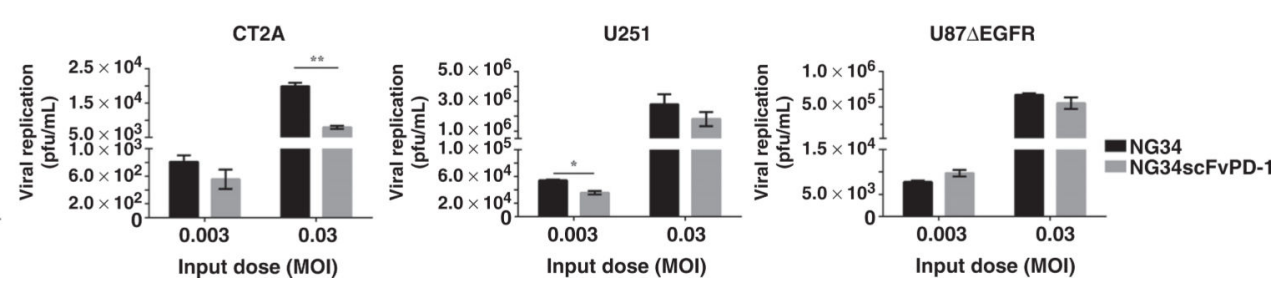

Figure 3.

In vitro evaluation of NG34 and NG34scFvPD-1 viral activity. A, GL261N4, CT2A, U251, and U87 $\triangle$ EGFR cells were infected at the indicated MOIs and cell survival was analyzed 6 days after infection by Sulforhodamine B assay. The viral lethal dose $50\left(\mathrm{LD}_{50}, \mathrm{pfu} / \mathrm{mL}\right)$ for each virus was calculated using a sigmoidal dose-response curve and is shown in the corresponding graph. The experiment shown is representative of experiments that were performed at least 3 times. B, GL261N4, CT2A, U251, and U87 $\triangle$ EGFR cells were infected at the indicated MOIs. After 72 hours, cells and supernatant were harvested and checked for viral replication using a plaque assay on Vero cells. Error bar, SEM. Group means were tested by unpaired $t$ test with Welch correction where * and ** indicate $P<0.05$ and $P<$ 0.01 , respectively $(n=3)$. 


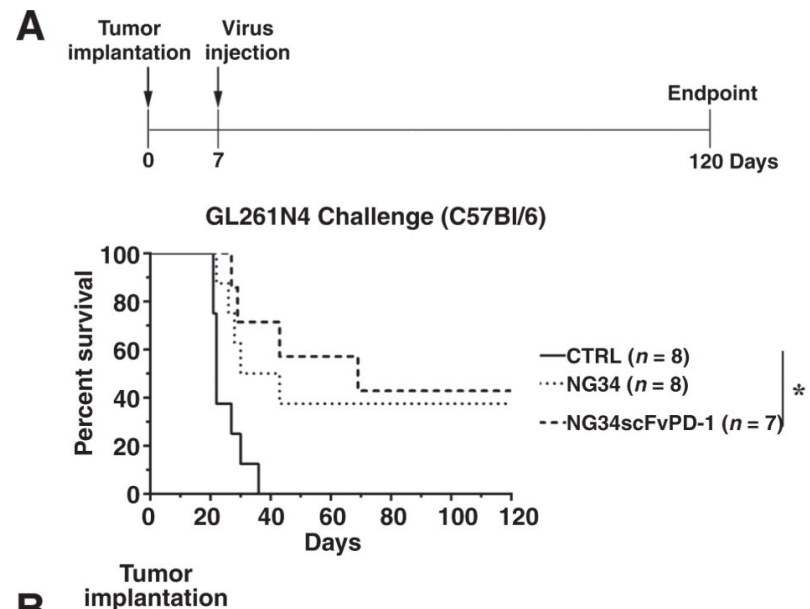

B implantation

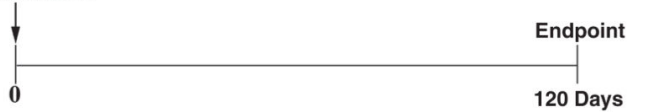

Rechallenge (C57BI/6)
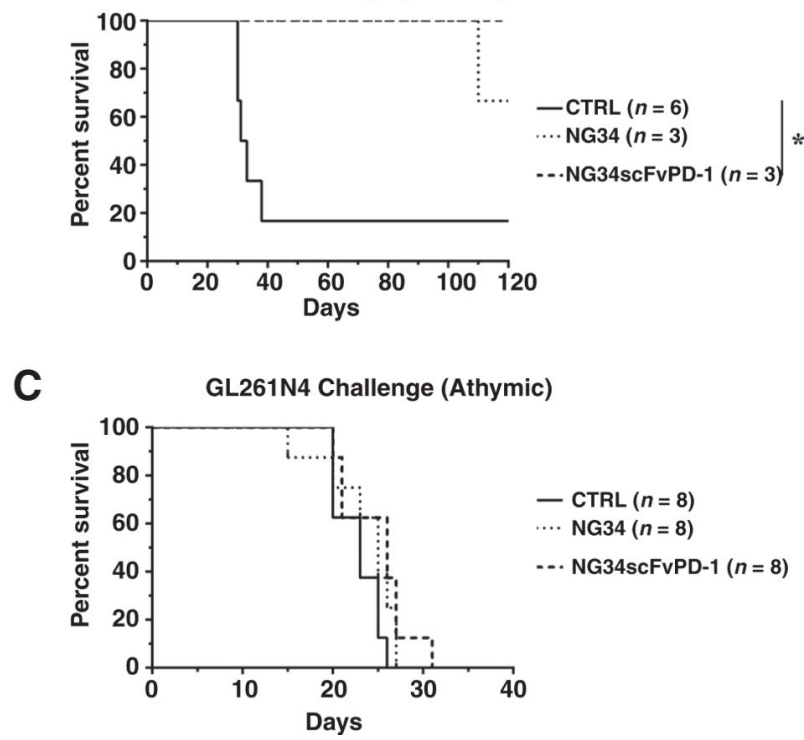

Figure 4.

In vivo evaluation of NG34scFvPD-1 antitumor efficacy. A, C57B1/6 mice were intracranially injected with GL261N4 cells ( $10^{5}$ cells). After 7 days, virus was intracranially administered at a dose of $1.5 \times 10^{6} \mathrm{pfu}$. Kaplan-Meier survival curve was used to show mice survival. *, $P<0.05$ (Mantel-Cox test and Holm post test). B, Long-term survivors (defined as mice surviving 120 days post-tumor inoculation) from the experiment in $\mathbf{A}$ were rechallenged with GL261N4 cells ( $10^{5}$ cells) in the contralateral brain hemisphere. Agematched $\mathrm{C} 57 \mathrm{Bl} / 6$ mice were used as controls. Kaplan-Meier survival curve was used to show mice survival. *, $P<0.05$; **, $P<0.01$ (Mantel-Cox test). C, Athymic mice were intracranially injected with GL261N4 cells ( $10^{5}$ cells). After 7 days, virus was intracranially administered at a dose of $1.5 \times 10^{6} \mathrm{pfu}$. Kaplan-Meier plot was used to show mice survival. Log-rank test was used to compare survival curves. 

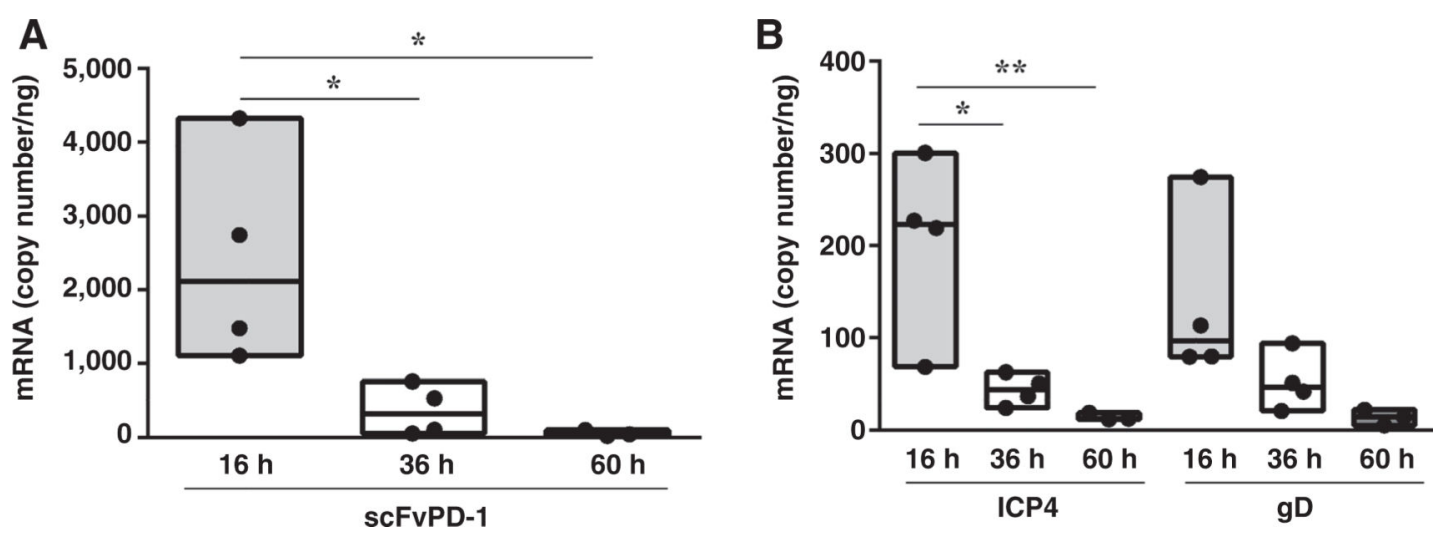

Figure 5.

In vivo viral gene and scFvPD-1 expression after GBM treatment with NG34scFvPD-1. C57Bl/6 mice were intracranially injected with GL261N4 cells ( $10^{5}$ cells). After 7 days, NG34scFvPD-1 was intracranially administered at a dose of $1.5 \times 10^{6} \mathrm{pfu} / \mathrm{mouse}$. Sixteen, 36, and 60 hours after virus inoculation, mice were sacrificed, their brains harvested, and RNA isolated. scFvPD-1 (A) and immediate early gene (ICP4) and late gene (gD) mRNA copy number were assessed by real-time PCR (B). One-way ANOVA (Tukey multiple comparisons test) was used for the analyses $(*, P<0.05 ; * *, P<0.01)$. 


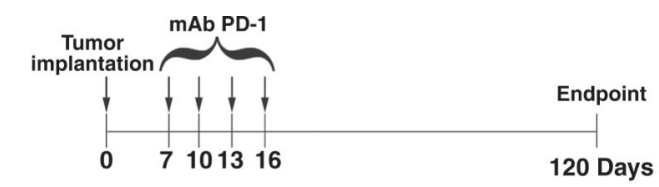

A

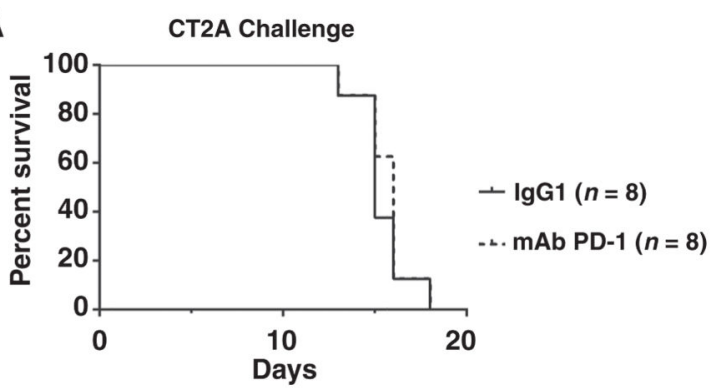

C implantation injection
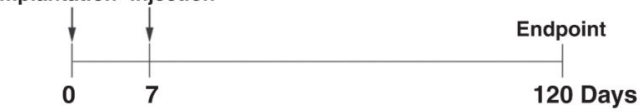

CT2A/PD-L1 Challenge

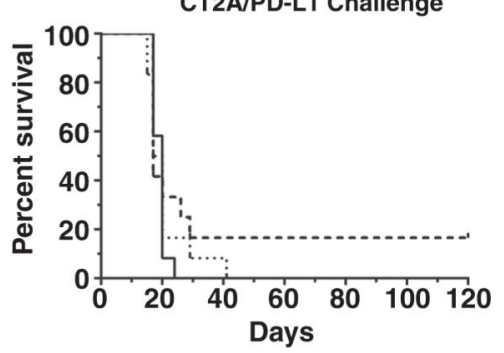

B
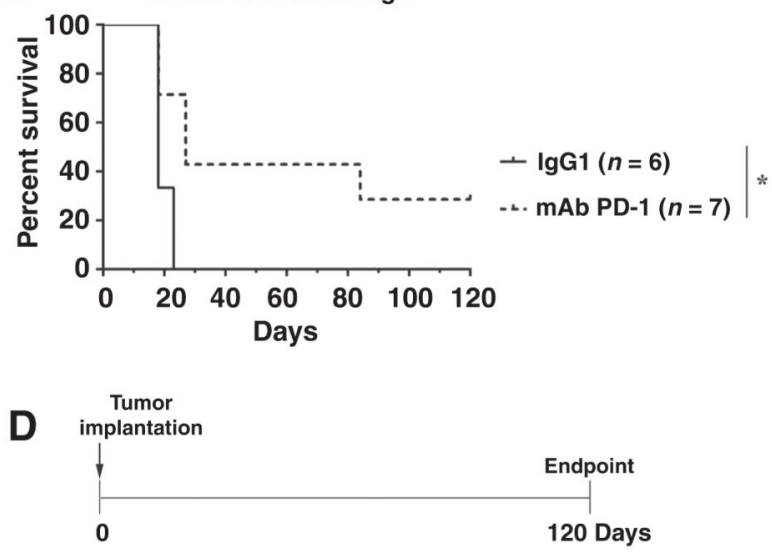

CT2A/PD-L1 Rechallenge

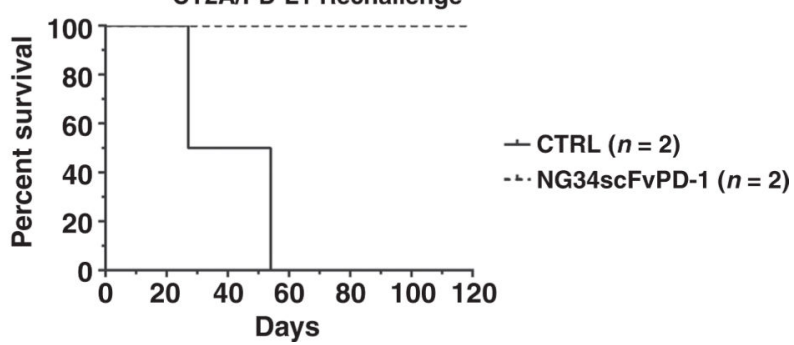

Figure 6.

In vivo evaluation of NG34scFvPD-1 antitumor efficacy. A, C57B1/6 mice were intracranially injected with CT2A cells $\left(4 \times 10^{5}\right.$ cells). After 7 days, the mAb PD- 1 antibody $(200 \mu \mathrm{g} /$ mouse) was intraperitoneally injected every 3 days for a total of 4 injections. Kaplan-Meier analysis was used to show animal survival. *, $P<0.05$ (Mantel-Cox test and Holm post-test). B, C57B1/6 mice were intracranially injected with CT2A cells overexpressing PD-L1 ( $4 \times 10^{5}$ cells). After 7 days, the mAb PD-1 antibody ( $200 \mathrm{mg} /$ mouse) was intraperitoneally injected every 3 days for a total of 4 injections. Kaplan-Meier analysis was used to show animal survival. *, $P<0.05$ (Mantel-Cox test and Holm posttest). C, C57Bl/6 mice were intracranially injected with CT2A cells overexpressing PD-L1 $\left(4 \times 10^{5}\right.$ cells). After 5 days, virus was intracranially administered at a dose of $1.5 \times 10^{6} \mathrm{pfu}$ Kaplan-Meier survival analysis5 was used to show animal survival. D, Long-term survivors of the experiment in B were rechallenged with CT2A cells overexpressing PD-L1 $\left(4 \times 10^{5}\right.$ cells) in contralateral brain hemisphere. Matched-age C57B1/6 mice have been used as control. Kaplan-Meier plot was used to show mice survival. Log-rank test was used to test differences between survival curves. 